\title{
Téoros
}

Revue de recherche en tourisme

\section{Un réseau de veille en tourisme}

\section{Paul Arseneault}

Volume 22, numéro 2, été 2003

URI : https://id.erudit.org/iderudit/1071599ar

DOI : https://doi.org/10.7202/1071599ar

Aller au sommaire du numéro

\section{Éditeur(s)}

Université du Québec à Montréal

\section{ISSN}

0712-8657 (imprimé)

1923-2705 (numérique)

Découvrir la revue

Citer cette note

Arseneault, P. (2003). Un réseau de veille en tourisme. Téoros, 22(2), 67-68. https://doi.org/10.7202/1071599ar

Ce document est protégé par la loi sur le droit d'auteur. L'utilisation des services d'Érudit (y compris la reproduction) est assujettie à sa politique d'utilisation que vous pouvez consulter en ligne.

https://apropos.erudit.org/fr/usagers/politique-dutilisation/
Cet article est diffusé et préservé par Érudit.

Érudit est un consortium interuniversitaire sans but lucratif composé de l’Université de Montréal, l'Université Laval et l'Université du Québec à Montréal. Il a pour mission la promotion et la valorisation de la recherche. https://www.erudit.org/fr/ 


\section{Cha ire de tourisme}

\section{Un réseau de veille en tourisme}

\section{Paul Arseneault}

a volatilité du tourisme, principalement en cette année de grande turbulence, interpelle les entreprises touristiques afin qu'elles développent une vision prospective et se positionnent avantageusement sur l'échiquier mondial. Dans ces circonstances, elles doivent effectuer une vigilance constante de l'environnement et avoir accès à l'information stratégique, ce qui correspond, concrètement, à exercer un processus de veille.

Le phénomène de veille demeure relativement récent non seulement au Québec, mais partout dans le monde. L'Organisation mondiale du tourisme et plusieurs gouvernements nationaux de différents pays ont appuyé la mise en place de pratiques de veille. À titre d'exemple, le ministère du Tourisme australien - dans son Green Paper (Department of Industry, Tourism and Resources, 2003 ) - souligne l'importance de la recherche pour l'amélioration de la compétitivité du secteur touristique alors que la France - dans un Livre Blanc ${ }^{1}$ - parle d'intelligence touristique et désigne la veille élargie et permanente comme un outil stratégique essentiel à l'ensemble des intervenants.

Au Québec, le mouvement de veille s'est initié au milieu des années 1990, lorsque le ministère de l'Industrie, du Commerce, de la Science et de la Technologie du Québec a créé un programme de support favorisant la création d'une quinzaine de centres de veille concurrentielle chargés d'aider les PME dans des secteurs d'activités aussi variés que la construction, la chimie et les télécommunications. De même, un nombre croissant de grandes entreprises privées et d'organismes d'État ont mis sur pied de tels services.

Néanmoins, malgré la pertinence de leurs actions, plusieurs de ces centres n'ont pas survécu. Certaines raisons expliquent leur disparition, dont:

- le manque de financement ;

- la difficulté à susciter l'intérêt des entreprises ;

- l'accent trop important mis sur les outils technologiques, mais insuffisant sur le contenu;

- la lourdeur découlant de la constitution des réseaux ou des processus administratifs.

Or, le contexte québécois a changé, notamment par les avancées technologiques qui rendent la veille plus flexible et par la prise de conscience, particulièrement dans l'industrie touristique, de l'importance de l'information prospective.

Depuis dix ans, la Chaire de Tourisme de l'Université du Québec à Montréal se positionne au cœur même de la recherche en tourisme par ses nombreux travaux qui touchent divers domaines tels que le transport aérien, le phénomène de concentration en tourisme, le cyclotourisme, la culture, les technologies et la saisonnalité. Désireuse de continuer à contribuer à l'essor et au rayonnement de l'industrie touristique, la Chaire travaille activement à implanter un réseau de veille en tourisme.

\section{Pourquoi une veille en tourisme?}

Plusieurs régions du Québec manifestent un intérêt croissant envers le tourisme comme secteur d'investissement et de développement de leur économie. Cependant, l'industrie touristique reste fragmentée et présente les caractéristiques suivantes:

- elle est constituée majoritairement de PME;

- elle regroupe une multitude d'organismes, d'associations, d'institutions et d'instances;

- ses ressources financières sont généralement limitées;

- ses dirigeants ont souvent des impératifs de vision à court terme;

- on observe une dispersion de l'industrie touristique au Québec avec ses multiples secteurs d'activités et produits touristiques;

- on note une forte concentration des compétences en tourisme dans le secteur privé.

La compétitivité d'une organisation est grandement liée à sa capacité d'adaptation et à sa rapidité de réaction. Cette adaptabilité exige qu'elle puisse anticiper des phénomènes grâce à la surveillance de l'environnement et à la collecte continue d'information. Se tenir à l'affût du changement de la nature et de l'intensité de la concurrence, de l'accélération des changements technologiques, de l'évolution des canaux de distribution et des nouveautés exige une veille constante. En outre, les entreprises sont trop souvent inondées d'information et doivent composer avec des données désuètes alors qu'elles ont besoin d'information analysée et pertinente.

Force est de constater que la valeur de l'information n'est pas stable dans le temps et qu'elle traverse différentes phases:

- elle décroît fortement avec le délai que le décideur est prêt à accorder pour la posséder (facteur de rapidité);

- elle croît fortement à la fois avec son exhaustivité et sa précision (facteur de synthèse);

- elle croît avec son influence sur une bonne prise de décisions;

- elle croît avec l'apport des informations « grises $^{2} »$.

La venue d'Internet permet désormais aux gestionnaires un accès à de plus en plus d'information, mais dont cette quantité même représente un élément rébarbatif. De plus, la fiabilité des sources s'avère souvent difficile à jauger et l'information trouvée sur Internet se présente souvent à un niveau d'analyse tel qu'il est souvent difficile, voire impossible, pour ces mêmes dirigeants d'en faire une synthèse qui permette une prise de décision éclairée.

Pour être valable aux yeux des gestionnaires touristiques, l'information doit: 
- catalyser sans dédoublement les données issues de différentes sources;

- valider de l'information contradictoire ou critique ;

- appliquer au contexte québécois les résultats d'études et d'expériences étrangères ;

- publier des données récentes et analysées (évolution et tendances);

- mettre à profit l'expertise de partenaires institutionnels, associatifs et privés aux échelles locale, nationale, et internationale pour l'obtention de sources d'information et l'enrichissement de l'information à diffuser.

\section{Un réseau de veille en tourisme}

Un réseau de veille en tourisme aura comme mandat de rester à l'affût de l'évolution de l'environnement dans lequel évolue l'industrie touristique québécoise, d'analyser l'information avec un regard prospectif, de l'enrichir auprès d'experts et de la diffuser promptement. Une des finalités de la veille visera à déceler, dans l'environnement des entreprises touristiques, les signes porteurs d'opportunités ou de menaces.

Un réseau de veille devra également se démarquer par son caractère analytique, par la richesse et la nouveauté de son information, par sa rapidité de production et de diffusion et par son souci d'offrir à l'industrie une information pratique, précise et accessible.

À partir d'un certain nombre de thématiques ciblées par un comité constitué d'organismes représentatifs des entreprises ainsi que des associations sectorielles et régionales, le réseau de veille en tourisme recueillera, traitera et analysera des renseignements pertinents. Les sources sont nombreuses : bases de données, enquêtes, études, revues spécialisées, colloques et conférences. Une fois colligés, les renseignements sur les thématiques retenues seront enrichis de l'avis d'experts et rapidement diffusés aux intervenants de l'industrie touristique québécoise par différents canaux: site portail, bulletins électroniques, chroniques dans des magazines spécialisés, etc.

\section{Une vision prospective}

Comme autre prémisse d'un réseau de veille efficace en tourisme, notons l'accent mis sur l'information prospective, c'est-à-dire celle qui permet d'envisager l'avenir, les grandes tendances qui le façonneront et les phénomènes qui auront un impact prévisible sur les entreprises et les secteurs de l'industrie touristique québécoise. À titre d'exemple, les événements extraordinaires de 2003 - guerre en Irak, SRAS auront eu des répercussions importantes sur la vitalité de l'industrie touristique mondiale, canadienne et québécoise : comment aurait-on pu minimiser ces impacts, quelles auraient été les meilleures stratégies pour les contrer, comment mesurer la reprise? Voilà autant de sujets d'intérêt pour un réseau de veille en tourisme.

Certains principes devront favoriser la pertinence de l'information colligée et traitée par un réseau de veille:

- ne pas diffuser uniquement des bilans du passé, mais plutôt en faire l'analyse pour en dégager des éléments prospectifs;

- juxtaposer les nombreuses sources d'information pour y déceler des tendances de fond;

- détecter des signes porteurs d'opportunités ou de menaces;

- trouver des façons d'innover;

- prévoir les changements.

Par ailleurs pour être porteuse, une veille doit se faire à temps plein, sur une base continue.

\section{Une approche réseau et une optique de collaboration}

Le rayon d'action d'un réseau de veille ne s'exerce pas là où des organismes ont déjà développé leur champ de compétence en matière de recherche ou d'étude spécifique. Loin de vouloir dupliquer le travail déjà réalisé par différents organismes qui font de la recherche (CCT, Tourisme Québec, Office du tourisme et des congrès de Québec, Tourisme Montréal, etc.), un réseau de veille devra plutôt être un trait d'union entre ces organisations, miser sur la collaboration de ces partenaires et tirer profit de leur expertise pour le bénéfice de tous.

Ainsi, un réseau de veille en tourisme ne devra pas se substituer aux organismes et aux entreprises qui font de la recherche, de la diffusion d'information ou qui offrent des services-conseils. Il devra plutôt collaborer avec ceux qui effectuent de la collecte de données pour mettre en place un processus de veille structuré, rapide et bonifié.

\section{La viabilité d'un réseau de veille}

Certaines conditions favoriseront l'implantation réussie et la pérennité d'un réseau de veille, assurant ainsi sa viabilité:

- réaliser un travail de sensibilisation pour inculquer une culture de veille au sein des PME québécoises et de leurs dirigeants;

- favoriser une collaboration et une synergie entre les organisations touristiques;

- bénéficier d'un support des pouvoirs publics, le projet relevant de l'intérêt commun de l'industrie;

- miser sur une représentativité des régions et des différents secteurs d'activités ;

- avoir recours à des veilleurs et à des experts impartiaux, objectifs, qualifiés et expérimentés qui ajouteront à la crédibilité du réseau ;

- favoriser la coopération avec plusieurs partenaires étrangers; on ne peut faire une veille «locale» à l'heure de la mondialisation;

- garantir la transparence de fonctionnement et la souplesse afin de répondre aux besoins changeants de l'industrie.

\section{Conclusion}

La Chaire de Tourisme est profondément convaincue de la pertinence et de la nécessité d'un réseau de veille en tourisme au Québec et elle est bien positionnée pour instaurer un tel projet en raison de son caractère impartial et de son rayonnement dans l'industrie.

Toutefois, faire de la veille relève de l'intérêt commun. Ainsi, la participation et le support des pouvoirs publics ainsi que la collaboration des organisations touristiques et des associations régionales et sectorielles sont des facteurs importants qui procureront les assises nécessaires à la Chaire de Tourisme pour aller de l'avant avec un tel projet.

Paul Arseneault est Directeur du Réseau de veille en tourisme, Chaire de Tourisme de l'UQAM.

\section{Notes}

1 Initiative de la Fédération Nationale des Comités départementaux du Tourisme (2003).

2 Information qui circule de manière informelle - rapports sectoriels, dossiers internes, notes de séminaires, études internes, etc.

\section{Bibliographie}

Department of Industry, Tourism and Resources, 2003, A Medium to Long Term Strategy for Tourism - Green Paper, Australie, 96 p.

Initiative de la Fédération Nationale des Comités départementaux du Tourisme, 2003, Livre Blanc - Tourisme \& Territoires, France, juin, $133 \mathrm{p}$. 